\title{
Factors influencing willingness to use fitness apps during COVID-19 pandemic: evidence from China
}

\author{
Jingyu Liu* \\ School of economics and management, Beijing Jiaotong University, China
}

\begin{abstract}
During the novel coronavirus pandemic, many people stopped going to the gym, and lack of exercise is likely to cause physical and mental health problems such as decreased immunity, in turn making them vulnerable to infection. Fitness apps can help people exercise at home by providing online professional guidance and supervision. This study explored the factors influencing fitness the intention to use apps during the epidemic in China. A new variable named epidemic crisis risk perception was added to the Unified Theory of Acceptance and Use of Technology model to reflect the impact of the epidemic. Performance expectation has the greatest impact on the willingness to use fitness apps. Therefore, developers must pay close attention to the needs of the public and improve the functions of apps to improve their satisfaction. In addition, the risk perception of epidemic crisis positively correlates with the willingness to use such apps, indicating that the novel coronavirus pandemic indeed affected public psychology and behavioural intention.
\end{abstract}

\section{Introduction}

In the novel coronavirus disease 2019 (COVID-19) pandemic, the number of people exercising in gyms decreased sharply. The reduction of exercise during the epidemic has begun to cause physical and mental health problems. To solve this problem, the World Health Organization has advocated sports activities at home [1]. Fitness apps are needed to offer guide including teaching videos, supervision and goal setting. Under this background, studying factors influencing willingness to use fitness apps has realistic meaning. It can provide suggestions for improving fitness apps and increasing the acceptance of fitness apps and thus promoting National Fitness. Besides, research also contributes to the development of theoretical understanding of public health and health apps.

Previous studies were mainly conducted in normal economic and social contexts. However, social psychology research shows that major public health events will alter public sensitivity, making their psychology and behaviour different from those of normal people [2]. For example, it is easy for people to refer to others as sources of information, resulting in herd psychology and herd behaviour [3]. Therefore, the previous research results and research methods are not fully applicable to the social situation in COVID-19. In the context of

*Corresponding author: 17753569959@163.com 
COVID-19, it is necessary to consider the social impact and psychological factors (such as people's perception of the risk level of the epidemic) in the analysis framework.

The main research questions of this study were: 1) What are the group characteristics of potential users? 2) What are the effects of fitness apps themselves and the social environment on users' willingness to use them? 3) What impact does COVID-19 have on the willingness to use fitness apps by changing public psychology?

The innovations and main contributions of this study are as follows. Firstly, a new potential variable termed epidemic crisis perceived risk (ECPR) was designed for the subjective assessment of infectious disease crisis risk, and the relationship between personal perception of the severity and harm of infectious diseases and the willingness to use health apps was analysed. Secondly, this study incorporated perceived cost, perceived risk, and other factors into the traditional Unified Theory of Acceptance and Use of Technology (UTAUT) model and more comprehensively considered the factors that may affect people's willingness to use fitness apps.

\section{Model introduction}

In 2003, Venkatesh et al. integrated previous theories and research models and proposed the UTAUT to analyse the factors influencing users' willingness and behaviour [4]. This model was developed based on the eight theories. The integration combines the advantages of all eight models. Therefore, the UTAUT model can explain the use behaviour and use intention with $70 \%$ accuracy, which is higher than that of the TAM. The corresponding model framework is shown in Figure 1.

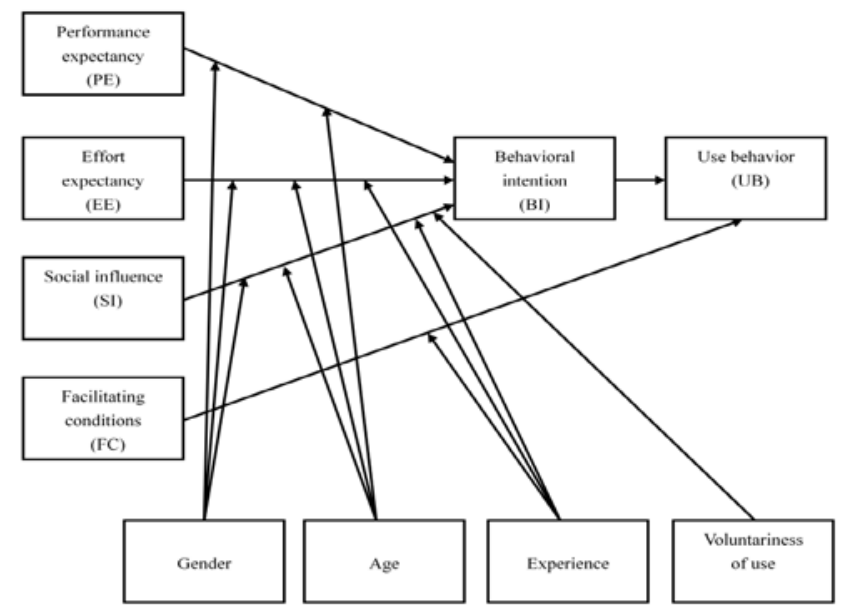

Fig. 1. Original UTAUT model.

\section{Research hypothesis}

To better explain the public's willingness to accept fitness apps and factors that influence the willingness under the epidemic situation, this study established a fitness app acceptance analysis model based on the UTAUT. Three variables of PC, PR, and ECPR were added to the original model to establish an extended UTAUT model. The overall framework of the research hypothesis and model is shown in Figure 2. 


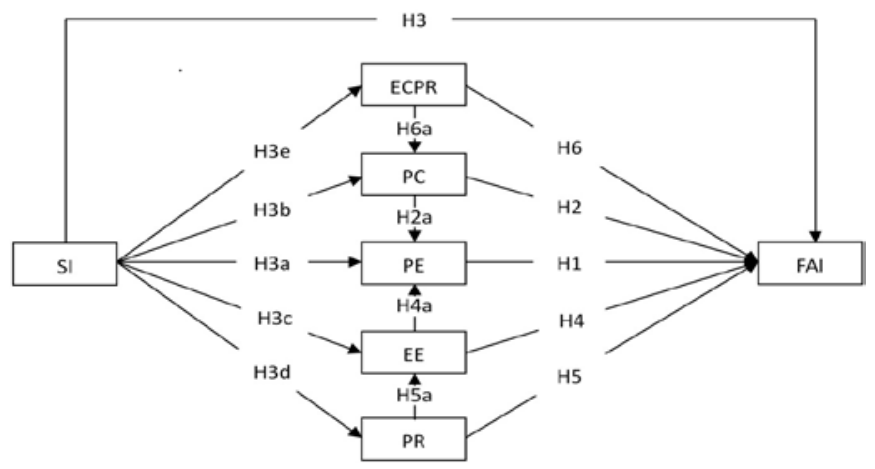

Fig. 2. Overall framework of the research hypothesis and model.

\section{Methods}

The respondents in this study were potential users(people who might have the of fitness apps. All the respondents who accepted the invitation to are voluntarily participate in the survey. A mobile-based questionnaire was used to collect information about the demographic characteristics, willingness to use fitness apps, and factors influencing the willingness. We used a 5-point Likert scale to measure each variable [5], and numbers 1 to 5 were chosen to express "highly disagree", "disagree", "uncertain", "agree", and "highly agree", respectively. This improved the total validity and discriminant validity of the questionnaire.

The survey was conducted simultaneously in all provinces of China between November and December 2020, and 780 formal questionnaires were distributed. After eliminating the invalid questionnaires, 711 valid questionnaires were collected; the effective recovery rate was $91.15 \%$. Demographic characteristics are shown in Table 1.

Table 1. Frequency Analysis Results.

\begin{tabular}{|c|c|c|c|c|}
\hline \multirow{2}{*}{ Name } & Option & $\begin{array}{c}\text { Frequency } \\
\square\end{array}$ & $\begin{array}{c}\text { Percentage(\%) } \\
\square\end{array}$ & $\begin{array}{c}\text { Cumulative } \\
\text { percentage(\%) }\end{array}$ \\
\hline \multirow{3}{*}{ Gender } & Male & 385 & 54.149 & 54.149 \\
\cline { 2 - 5 } & Female & 326 & 45.851 & 100 \\
\hline \multirow{4}{*}{ Age } & $<30$ & 320 & 45.007 & 45.007 \\
\cline { 2 - 5 } & $30-40$ & 224 & 31.505 & 76.512 \\
\cline { 2 - 5 } & $40-50$ & 113 & 15.893 & 92.405 \\
\cline { 2 - 5 } Educational \\
Level & $>50$ & 54 & 7.595 & 100 \\
\cline { 2 - 5 } & $\begin{array}{c}\text { Senior high school } \\
\text { and below }\end{array}$ & 97 & 13.643 & 13.643 \\
\cline { 2 - 5 } & Undergraduate & 523 & 73.558 & 87.201 \\
\hline \multirow{3}{*}{$\begin{array}{c}\text { Working } \\
\text { Status }\end{array}$} & Fubove & 91 & 12.799 & 100 \\
\cline { 2 - 5 } & Part-time & 425 & 59.775 & 59.775 \\
\cline { 2 - 5 } & Students & 189 & 13.643 & 100 \\
\hline \multirow{4}{*}{ Income } & $<3000$ yuan/month & 376 & 52.582 & 52.883 \\
\cline { 2 - 5 } & $\begin{array}{c}3000-6000 \\
\text { yuan/month }\end{array}$ & 178 & 25.035 & 77.918 \\
\cline { 2 - 5 } & $\begin{array}{c}6000-9000 \\
\text { yuan/month }\end{array}$ & 83 & 11.674 & 89.592 \\
\cline { 2 - 5 } & $>9000$ yuan/month & 74 & 10.408 & 100 \\
\hline & Total & 711 & 100 & 100 \\
\hline
\end{tabular}




\section{Results}

\subsection{Reliability analysis}

This study used Cronbach's alpha coefficient as the test standard to observe the consistency of each item of the questionnaire. It is generally believed that when Cronbach's alpha is greater than 0.7 , the measurement model has good internal consistency. The results showed that the overall Cronbach's alpha coefficient of the scale was greater than 0.7 , and so was the Cronbach's alpha coefficient of the seven dimensions, indicating that the internal consistency of the questionnaire was good; therefore, we can consider the reliability of the survey results to be excellent. In the reliability test, items that had a correlation coefficient with the whole dataset of less than 0.3 were identified so that they could be deleted. Finally, the correlation between the items in the questionnaire and the overall score was higher than 0.3 (Table 2), indicating that most of the items are well correlated with the overall score and have discrimination power.

Table 2. Reliability analysis of the questionnaire.

\begin{tabular}{|c|c|c|c|c|c|}
\hline & $\begin{array}{l}\text { Scale mean } \\
\text { after Item } \\
\text { Deletion }\end{array}$ & $\begin{array}{l}\text { Scale variance } \\
\text { after Item } \\
\text { Deletion }\end{array}$ & $\begin{array}{l}\text { Corrected } \\
\text { item total } \\
\text { correlation }\end{array}$ & $\begin{array}{c}\text { Cronbach's } \\
\text { alpha value after } \\
\text { Item Deletion }\end{array}$ & $\begin{array}{c}\text { Cronbach's } \\
\text { Alpha }\end{array}$ \\
\hline PE1 & 10.326 & 8.584 & 0.641 & 0.811 & \multirow{4}{*}{0.840} \\
\hline PE2 & 10.315 & 8.425 & 0.671 & 0.798 & \\
\hline PE3 & 10.038 & 7.634 & 0.726 & 0.773 & \\
\hline PE4 & 10.325 & 8.606 & 0.656 & 0.805 & \\
\hline EE1 & 6.899 & 4.762 & 0.658 & 0.767 & \multirow{3}{*}{0.819} \\
\hline EE2 & 6.869 & 4.872 & 0.646 & 0.778 & \\
\hline EE3 & 6.648 & 3.913 & 0.725 & 0.699 & \\
\hline SI1 & 6.878 & 4.995 & 0.675 & 0.790 & \multirow{3}{*}{0.834} \\
\hline SI2 & 6.616 & 3.975 & 0.735 & 0.734 & \\
\hline SI3 & 6.897 & 4.858 & 0.686 & 0.778 & \\
\hline PC1 & 5.398 & 4.026 & 0.727 & 0.726 & \multirow{3}{*}{0.829} \\
\hline PC2 & 5.121 & 4.864 & 0.673 & 0.777 & \\
\hline PC3 & 5.093 & 4.952 & 0.671 & 0.780 & \\
\hline PR1 & 5.056 & 4.788 & 0.664 & 0.776 & \multirow{3}{*}{0.825} \\
\hline PR2 & 5.360 & 3.893 & 0.732 & 0.708 & \\
\hline PR3 & 5.060 & 4.843 & 0.658 & 0.781 & \\
\hline ECPR1 & 6.800 & 4.760 & 0.666 & 0.767 & \multirow{3}{*}{0.822} \\
\hline ECPR2 & 6.805 & 4.617 & 0.678 & 0.754 & \\
\hline ECPR3 & 6.567 & 3.913 & 0.697 & 0.740 & \\
\hline FAI1 & 7.037 & 4.621 & 0.665 & 0.754 & \multirow{3}{*}{0.817} \\
\hline FAI2 & 6.795 & 3.938 & 0.708 & 0.713 & \\
\hline FAI3 & 6.987 & 4.827 & 0.646 & 0.774 & \\
\hline
\end{tabular}

\subsection{Exploratory factor analysis}

The text of your paper should be formatted as follows:

Table 3 shows that the publicity value of all research projects is greater than 0.4 , which indicates that the research project information can be effectively extracted. In addition, the KMO value was 0.867 , greater than 0.6 , indicating that the data are valid. The variance explanation rates of the seven factors were $12.550 \%, 10.356 \%, 10.325 \%, 10.226 \%, 10.182 \%$, $10.134 \%$, and $10.014 \%$, and the cumulative variance explanation rate after rotation was $73.788 \%$, which is $>60 \%$. 
Table 3. Results of validity analysis.

\begin{tabular}{|c|c|c|c|c|c|c|c|c|}
\hline \multirow[b]{2}{*}{ Name } & \multicolumn{7}{|c|}{ Load Factor $\square$} & \multirow{2}{*}{$\begin{array}{c}\text { Common } \\
\text { degree } \\
\text { (common } \\
\text { factor } \\
\text { variance) } \\
\square\end{array}$} \\
\hline & Factor1 & Factor2 & Factor3 & Factor4 & Factor5 & $\begin{array}{l}\text { Factor } \\
6\end{array}$ & Factor7 & \\
\hline PE1 & 0.757 & & & & & & & 0.644 \\
\hline PE2 & 0.752 & & & & & & & 0.677 \\
\hline PE3 & 0.874 & & & & & & & 0.78 \\
\hline PE4 & 0.754 & & & & & & & 0.656 \\
\hline EE1 & & & & & 0.777 & & & 0.711 \\
\hline EE2 & & & & & 0.772 & & & 0.696 \\
\hline EE3 & & & & & 0.878 & & & 0.81 \\
\hline SI1 & & & 0.769 & & & & & 0.72 \\
\hline SI2 & & & 0.891 & & & & & 0.822 \\
\hline $\mathrm{SI} 3$ & & & 0.807 & & & & & 0.747 \\
\hline PC1 & & 0.886 & & & & & & 0.809 \\
\hline $\mathrm{PC} 2$ & & 0.803 & & & & & & 0.727 \\
\hline PC3 & & 0.794 & & & & & & 0.722 \\
\hline PR1 & & & & 0.784 & & & & 0.713 \\
\hline PR2 & & & & 0.885 & & & & 0.818 \\
\hline PR3 & & & & 0.787 & & & & 0.712 \\
\hline ECPR1 & & & & & & 0.763 & & 0.714 \\
\hline ECPR2 & & & & & & 0.801 & & 0.735 \\
\hline ECPR3 & & & & & & 0.861 & & 0.785 \\
\hline FAI1 & & & & & & & 0.787 & 0.731 \\
\hline FAI2 & & & & & & & 0.874 & 0.802 \\
\hline FAI3 & & & & & & & 0.769 & 0.701 \\
\hline $\begin{array}{c}\text { Eigenvalue value } \\
\text { (before rotation) } \square\end{array}$ & 6.75 & 1.929 & 1.667 & 1.591 & 1.499 & 1.447 & 1.352 & - \\
\hline $\begin{array}{c}\text { Variance } \\
\text { explanation rate } \\
\text { (before rotation) } \square\end{array}$ & $\begin{array}{c}30.68 \\
\%\end{array}$ & $8.77 \%$ & $7.58 \%$ & $7.23 \%$ & $6.81 \%$ & $6.58 \%$ & $6.14 \%$ & - \\
\hline $\begin{array}{c}\text { Cumulative variance } \\
\text { explanation } \\
\text { rate(before } \\
\text { rotation) } \square\end{array}$ & $\begin{array}{c}30.68 \\
\%\end{array}$ & $\begin{array}{c}39.45 \\
\%\end{array}$ & $\begin{array}{c}47.02 \\
\%\end{array}$ & $\begin{array}{c}54.25 \\
\%\end{array}$ & $\begin{array}{c}61.07 \\
\%\end{array}$ & $\begin{array}{c}67.65 \\
\%\end{array}$ & $73.79 \%$ & - \\
\hline $\begin{array}{l}\text { Eigenvalue value } \\
\text { (after rotation) } \square\end{array}$ & 2.761 & 2.278 & 2.272 & 2.25 & 2.24 & 2.23 & 2.203 & - \\
\hline $\begin{array}{c}\text { Variance } \\
\text { explanation rate } \\
\text { (after rotation) }\end{array}$ & $\begin{array}{c}12.55 \\
\%\end{array}$ & $\begin{array}{c}10.36 \\
\%\end{array}$ & $\begin{array}{c}10.33 \\
\%\end{array}$ & $\begin{array}{c}10.23 \\
\%\end{array}$ & $\begin{array}{c}10.18 \\
\%\end{array}$ & $\begin{array}{c}10.13 \\
\%\end{array}$ & $10.01 \%$ & - \\
\hline $\begin{array}{c}\text { Cumulative variance } \\
\text { explanation rate } \\
\text { (after rotation) }\end{array}$ & $\begin{array}{c}12.55 \\
\%\end{array}$ & $\begin{array}{c}22.91 \\
\%\end{array}$ & $\begin{array}{c}33.23 \\
\%\end{array}$ & $\begin{array}{c}43.46 \\
\%\end{array}$ & $\begin{array}{c}53.64 \\
\%\end{array}$ & $\begin{array}{c}63.77 \\
\%\end{array}$ & $73.79 \%$ & - \\
\hline KMO value $\square$ & & & & 0.867 & & & & - \\
\hline $\begin{array}{c}\text { Barthes spherical } \\
\text { value } \square\end{array}$ & & & & 7045.26 & & & & - \\
\hline $\mathrm{df} \square$ & & & & 231 & & & & - \\
\hline $\mathrm{p}$ value $\square$ & & & & 0 & & & & - \\
\hline
\end{tabular}

According to the meaning of the items in the scale and the rotating component matrix, if the load value is greater than 0.5 , it can be analysed as an important item. As shown in Table 3 , the load value of each item in each dimension was greater than 0.5 . At the same time, the results of the rotating component matrix were consistent with the scale and dimensions divided by the research design. Therefore, the validity of the questionnaire was considered to be high, and the questionnaire was effective. 


\subsection{Confirmatory factor analysis}

In the research model of the present study, CMIN/DF, NFI, IFI, TLI, CFI, GFI, RMSEA, CFI, and other model fitness indicators met the standards, indicating very good model fitness (Table 4).

Table 4. Model fitness index.

\begin{tabular}{|c|c|c|c|c|c|c|c|c|}
\hline CMIN & df & CMIN/DF & NFI & IFI & TLI & CFI & GFI & RMSEA \\
\hline 417.646 & 188.000 & 2.222 & 0.934 & 0.963 & 0.954 & 0.962 & 0.951 & 0.041 \\
\hline Suggested value & & $<3$ & $>0.8$ & $>0.9$ & $>0.8$ & $>0.9$ & $>0.8$ & $<0.08$ \\
\hline
\end{tabular}

In this study, construct reliability (CR) and average variance extracted (AVE) are used to evaluate the convergence effectiveness. When the CR value of each factor is greater than 0.7 and the AVE value is greater than 0.50, it generally indicates good convergence efficiency. In addition, when the square root value of the AVE of each factor is greater than the correlation coefficient between the factor and other factors, the discriminant validity is higher. The basic AVE of each dimension was greater than 0.5 , and the CR value was greater than 0.7 , indicating that the convergence validity of this dimension was high (Table 5 and Table $6)$.

Table 5. Convergence validity.

\begin{tabular}{|c|c|c|c|c|c|c|c|c|c|}
\hline \multicolumn{2}{|c|}{ Variable Relation } & $\begin{array}{c}\text { Coefficient } \\
\text { Estimate }\end{array}$ & $\begin{array}{c}\text { Standard } \\
\text { Error }\end{array}$ & $\begin{array}{c}\text { Critical } \\
\text { Value }\end{array}$ & P & $\begin{array}{c}\text { Factor } \\
\text { Loading }\end{array}$ & AVE & CR \\
\hline SI1 & $<---$ & SI & 1.000 & & & & 0.760 & 0.609 & 0.824 \\
\hline SI2 & $<---$ & SI & 1.274 & 0.067 & 18.927 & $* * *$ & 0.810 & & \\
\hline SI3 & $<---$ & SI & 1.034 & 0.056 & 18.515 & $* * *$ & 0.770 & & \\
\hline ECPR1 & $<---$ & ECPR & 1.000 & & & & 0.761 & 0.581 & 0.806 \\
\hline ECPR2 & $<---$ & ECPR & 1.025 & 0.058 & 17.670 & $* * *$ & 0.761 & & \\
\hline ECPR3 & $<---$ & ECPR & 1.185 & 0.067 & 17.712 & $* * *$ & 0.765 & & \\
\hline PC1 & $<---$ & PC & 1.000 & & & & 0.810 & 0.602 & 0.819 \\
\hline PC2 & $<---$ & PC & 0.815 & 0.044 & 18.572 & $* * *$ & 0.760 & & \\
\hline PC3 & $<---$ & PC & 0.791 & 0.043 & 18.523 & $* * *$ & 0.756 & & \\
\hline EE1 & $<---$ & EE & 1.000 & & & & 0.748 & 0.581 & 0.806 \\
\hline EE2 & $<---$ & EE & 0.952 & 0.056 & 17.049 & $* * *$ & 0.725 & & \\
\hline EE3 & $<---$ & EE & 1.248 & 0.070 & 17.904 & $* * *$ & 0.811 & & \\
\hline PR1 & $<---$ & PR & 1.000 & & & & 0.747 & 0.589 & 0.811 \\
\hline PR2 & $<---$ & PR & 1.261 & 0.070 & 18.061 & $* * *$ & 0.815 & & \\
\hline PR3 & $<---$ & PR & 0.978 & 0.056 & 17.379 & $* * *$ & 0.738 & & \\
\hline FAI1 & $<---$ & FAI & 1.000 & & & & 0.765 & 0.584 & 0.808 \\
\hline FAI2 & $<---$ & FAI & 1.183 & 0.066 & 18.056 & $* * *$ & 0.793 & & \\
\hline FAI3 & $<---$ & FAI & 0.935 & 0.054 & 17.397 & $* * *$ & 0.734 & & \\
\hline PE1 & $<---$ & PE & 1.000 & & & & 0.688 & 0.537 & 0.823 \\
\hline PE2 & $<---$ & PE & 1.098 & 0.066 & 16.727 & $* * *$ & 0.752 & & \\
\hline PE3 & $<---$ & PE & 1.232 & 0.072 & 17.031 & $* * *$ & 0.773 & & \\
\hline PE4 & $<---$ & PE & 1.020 & 0.063 & 16.115 & $* * *$ & 0.716 & & \\
\hline
\end{tabular}

\subsection{Correlation analysis}

Correlation analysis was used to study the correlation between the willingness to use fitness apps and PE, EE, SI, PC, PR, and ECPR, and the Pearson correlation coefficient was used to express the strength of the correlation (Table 7). Willingness to use fitness apps correlated 
with PE (0.352; P < 0.01), EE (0.316; P < 0.01), SI (0.318; P <0.01), PC (-0.288; P < 0.01), PR (-0.309; P $<0.01)$, and ECPR (0.328; P $<0.01)$.

Table 6. Discriminant validity.

\begin{tabular}{|c|c|c|c|c|c|c|c|c|}
\hline \multicolumn{2}{|c|}{} & SI & ECPR & PC & EE & PR & FAI & PE \\
\hline SI & $\begin{array}{c}\text { Pearson } \\
\text { Correlation }\end{array}$ & 0.780 & & & & & & \\
\hline ECPR & $\begin{array}{c}\text { Pearson } \\
\text { Correlation }\end{array}$ & $.352^{* *}$ & 0.762 & & & & & \\
\hline PC & $\begin{array}{c}\text { Pearson } \\
\text { Correlation }\end{array}$ & $-.258^{* *}$ & $-.334^{* *}$ & 0.776 & & & & \\
\hline EE & $\begin{array}{c}\text { Pearson } \\
\text { Correlation }\end{array}$ & $.296^{* *}$ & $.346^{* *}$ & $-.288^{* *}$ & 0.762 & & & \\
\hline FAI & $\begin{array}{c}\text { Pearson } \\
\text { Correlation }\end{array}$ & $-.275^{* *}$ & $-.301^{* *}$ & $.308^{* *}$ & $-.367^{* *}$ & 0.767 & & \\
\hline Pearson & $.318^{* *}$ & $.328^{* *}$ & $-.288^{* *}$ & $.316^{* *}$ & $-.309^{* *}$ & 0.764 & \\
\hline $\begin{array}{c}\text { Pearson } \\
\text { Correlation }\end{array}$ & $.345^{* *}$ & $.328^{* *}$ & $-.259^{* *}$ & $.327^{* *}$ & $-.283^{* *}$ & $.352^{* *}$ & 0.733 \\
\hline
\end{tabular}

Table 7. Pearson correlation.

\begin{tabular}{|c|c|c|c|c|c|c|c|c|c|}
\hline & $\begin{array}{l}\text { Ave } \\
\text { rage } \\
\text { Val } \\
\text { ue }\end{array}$ & $\begin{array}{c}\text { Standa } \\
\text { rd } \\
\text { deviati } \\
\text { on }\end{array}$ & FAI & PE & $\mathrm{EE}$ & SI & PC & PR & $\begin{array}{c}\text { ECP } \\
\mathrm{R}\end{array}$ \\
\hline FAI & 3.47 & 1.011 & 1 & & & & & & \\
\hline $\mathrm{PE}$ & $\begin{array}{c}3.41 \\
7\end{array}$ & 0.936 & $\begin{array}{c}0.352^{*} \\
* *\end{array}$ & 1 & & & & & \\
\hline $\mathrm{EE}$ & $\begin{array}{c}3.40 \\
3 \\
\end{array}$ & 1.017 & $\begin{array}{c}0.316^{*} \\
* *\end{array}$ & $\begin{array}{c}0.327 * \\
* *\end{array}$ & 1 & & & & \\
\hline SI & $\begin{array}{c}3.39 \\
9 \\
\end{array}$ & 1.032 & $\begin{array}{c}0.318^{*} \\
* *\end{array}$ & $\begin{array}{c}0.345^{*} \\
* *\end{array}$ & $\begin{array}{c}0.296^{*} \\
* *\end{array}$ & 1 & & & \\
\hline $\mathrm{PC}$ & $\begin{array}{c}2.60 \\
2\end{array}$ & 1.031 & $\begin{array}{c}- \\
0.288^{*} \\
* *\end{array}$ & $\begin{array}{c}- \\
0.259 * \\
* *\end{array}$ & $\begin{array}{c}- \\
0.288^{*} \\
* *\end{array}$ & $\begin{array}{c}- \\
0.258^{*} \\
* *\end{array}$ & 1 & & \\
\hline PR & $\begin{array}{c}2.57 \\
9\end{array}$ & 1.018 & $\begin{array}{c}- \\
0.309^{*} \\
* *\end{array}$ & $\begin{array}{c}- \\
0.283 * \\
* *\end{array}$ & $\begin{array}{c}- \\
0.367 * \\
* *\end{array}$ & $\begin{array}{c}- \\
0.275^{*} \\
* *\end{array}$ & $\begin{array}{c}0.308^{*} \\
* *\end{array}$ & 1 & \\
\hline $\begin{array}{c}\text { ECP } \\
\text { R }\end{array}$ & $\begin{array}{c}3.36 \\
2\end{array}$ & 1.009 & $\begin{array}{c}0.328^{*} \\
* *\end{array}$ & $\begin{array}{c}0.328 * \\
* *\end{array}$ & $\begin{array}{c}0.346^{*} \\
* *\end{array}$ & $\begin{array}{c}0.352^{*} \\
* *\end{array}$ & $\begin{array}{c}- \\
0.334 * \\
* *\end{array}$ & $\begin{array}{c}- \\
0.301 * \\
* *\end{array}$ & 1 \\
\hline
\end{tabular}

\subsection{Modelling and analysis}

In many social science, finance, psychology, and management studies, many potential variables cannot be directly observed, such as learning motivation and user satisfaction; the traditional model cannot deal with these variables, whereas the structural equation model can $[6,7,8]$. Therefore, structural equation modelling was used to analyse the data (Figure 3). 


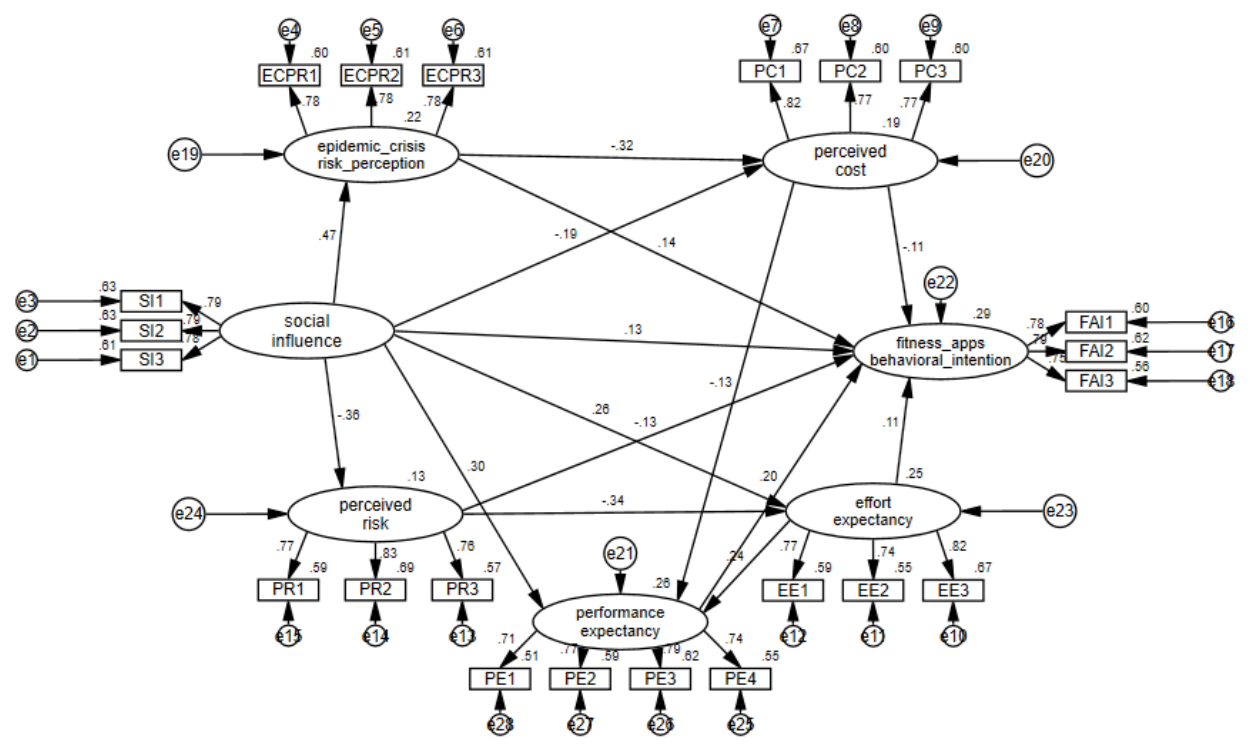

Fig. 3. Results of the structural equation modelling.

In this research model, CMIN/DF, NFI, IFI, TLI, CFI, GFI, RMSEA, CFI, and other model fitness indicators met the standard, indicating very good model fitness (Table 8).

Table 8. Model fitness index.

\begin{tabular}{|c|c|c|c|c|c|c|c|c|}
\hline CMIN & df & CMIN/DF & NFI & IFI & TLI & CFI & GFI & RMSEA \\
\hline 563.054 & 194.000 & 2.902 & 0.921 & 0.947 & 0.936 & 0.946 & 0.934 & 0.052 \\
\hline Suggested value & & $<3$ & $>0.8$ & $>0.9$ & $>0.8$ & $>0.9$ & $>0.8$ & $<0.08$ \\
\hline
\end{tabular}

Table 9. Path test results.

\begin{tabular}{|c|c|c|c|c|c|c|c|c|c|}
\hline Path & \multicolumn{2}{|c|}{$\begin{array}{c}\text { Relationship Path } \\
\text { Between Variables }\end{array}$} & $\begin{array}{c}\text { Nonstandard } \\
\text { Regression } \\
\text { Coefficient }\end{array}$ & $\begin{array}{c}\text { Standardized } \\
\text { Regression } \\
\text { Coefficient }\end{array}$ & $\begin{array}{c}\text { Standard } \\
\text { Error }\end{array}$ & $\begin{array}{c}\text { T } \\
\text { Value }\end{array}$ & P & $\begin{array}{c}\text { Path } \\
\text { Test } \\
\text { Resu } \\
\text { lts }\end{array}$ \\
\hline Path 1 & ECPR & $<---$ & SI & 0.449 & 0.466 & 0.045 & 10.027 & $* * *$ & For \\
\hline Path 2 & PR & $<---$ & SI & -0.344 & -0.360 & 0.044 & -7.840 & $* * *$ & For \\
\hline Path 3 & PC & $<---$ & SI & -0.227 & -0.188 & 0.060 & -3.752 & $* * *$ & For \\
\hline Path 4 & EE & $<---$ & SI & 0.316 & 0.262 & 0.056 & 5.692 & $* * *$ & For \\
\hline Path 5 & PC & $<---$ & $\begin{array}{c}\text { EC } \\
\text { PR }\end{array}$ & -0.400 & -0.319 & 0.065 & -6.183 & $* * *$ & For \\
\hline Path 6 & EE & $<---$ & PR & -0.431 & -0.342 & 0.060 & -7.233 & $* * *$ & For \\
\hline Path 7 & PE & $<---$ & SI & 0.278 & 0.304 & 0.046 & 6.051 & $* * *$ & For \\
\hline Path8 & PE & $<---$ & PC & -0.100 & -0.133 & 0.033 & -3.004 & $\begin{array}{c}0.00 \\
3\end{array}$ & For \\
\hline Path9 & PE & $<---$ & EE & 0.178 & 0.235 & 0.035 & 5.067 & $* * *$ & For \\
\hline Path10 & FAI & $<---$ & SI & 0.133 & 0.134 & 0.057 & 2.339 & $\begin{array}{c}0.01 \\
9\end{array}$ & For \\
\hline Path11 & FAI & $<---$ & $\begin{array}{c}\text { EC } \\
\text { PR }\end{array}$ & 0.143 & 0.138 & 0.053 & 2.706 & $\begin{array}{c}0.00 \\
7\end{array}$ & For \\
\hline Path 12 & FAI & $<--$ & PC & -0.094 & -0.114 & 0.039 & -2.424 & $\begin{array}{c}0.01 \\
5\end{array}$ & For \\
\hline Path 13 & FAI & $<---$ & PE & 0.218 & 0.200 & 0.054 & 4.057 & $* * *$ & For \\
\hline Path 14 & FAI & $<---$ & EE & 0.092 & 0.111 & 0.042 & 2.193 & $\begin{array}{c}0.02 \\
8\end{array}$ & For \\
\hline Path 15 & FAI & $<---$ & PR & -0.134 & -0.129 & 0.050 & -2.667 & $\begin{array}{c}0.00 \\
8\end{array}$ & For \\
\hline
\end{tabular}


Based on the structural model path coefficient, the research hypotheses of paths 1 through 15 were verified (Table 10). There was a significant positive impact between SI and ECPR ( $\beta=0.466, P<0.05$ ), supporting hypothesis path 1 ; there was a significant negative impact between SI and PR $(\beta=-0.360, \mathrm{P}<0.05)$, supporting hypothesis path 2 ; and there was a significant negative impact between SI and PC ( $\beta=-0.188, \mathrm{P}<0.05)$, supporting hypothesis path 3. Similarly, data supporting hypothesis paths 4 through 15 are shown in the table.

\section{Conclusions}

Fitness apps can largely replace fitness coaches to provide services for people who exercise at home or other private places during epidemics. The purpose of this study was to explore the factors that affect the willingness of people to use fitness apps. The results showed that $\mathrm{PE}$ is still the most important factor $[9,10]$, and ECRP also has a significant impact on the willingness to use fitness apps. A high willingness closely relates to the people's high perception of the epidemic risk and crisis. The results of this study enhance our understanding of the intention to use fitness apps under an epidemic situation and reveal the impact of an epidemic on people's behaviour intention amidst the changing social atmosphere and public psychology. At a theoretical level, this study provides a reference for future research on fitness app use intention during major public health events. Understanding the obvious influencing factors can also guide the promotion of fitness apps in practice.

\section{References}

1. World Health Organization. \#HealthyAtHome-Physical activity. Available online: https://www.who.int/news-room/campaigns/connecting-the-world-to-combatcoronavirus/healthyathome/healthyathome---physical-activity (accessed on 15 May 2020).

2. H. Chen, K.Zhang Insight into the psychological problems on the epidemic of COVID19 in China by online searching behaviors. J Affect Disord;276,1093-1094.(2020)

3. Lee $\mathrm{YC}, \mathrm{Wu} \mathrm{WL}$, Lee CK. How COVID-19 triggers our herding behavior? Risk perception, state anxiety, and trust. Front Public Health,9 (2021)

4. Venkatesh V, Morris MG, Davis GB, Davis FD. User acceptance of information technology: Toward a unified view. MIS Quarterly,27,425-478(2003)

5. Dawes J. Do data characteristics change according to the number of scale points used? An experiment using 5-point, 7-point and 10-point scales. Int J Market Res,50,6177(2008)

6. Hoogland JJ, Boomsma A. Robustness studies in covariance structure modeling - An overview and a meta-analysis. Sociol Methods Res,26,329-367(1998)

7. Kline RB. Response to Leslie Hayduk's review of principles and practice of structural equation modeling, 4th edition. Can Stud Popul,45,188-195(2008)

8. Priester JR. The use of structural equation models in consumer psychology: A methodological dialogue on its contributions, cautions, and concerns. J Consumer Psychol,20,205-207,(2010)

9. Cho J, Lee HE, Quinlan M. Cross-national comparisons of college students' attitudes toward diet/fitness apps on smartphones. J Am Coll Health,65,437-449(2017)

10. Davis FD. Perceived usefulness, perceived ease of use, and user acceptance of information technology. MIS Quarterly,13,319-340(1989) 